\title{
Adverse reactions and safety profile of the mRNA COVID-19 vaccines among Asian military personnel
}

Joshua TC $\underline{\text { Tan }}{ }^{1,2}{ }_{M B B S}$, Clive Tan ${ }^{1,3}{ }_{M B B S}$, Jeremy Teoh ${ }^{1,4}{ }_{M B B S}$, Wahab MT ${ }^{1,5}{ }_{M B B S}$, Guan Zhong $\underline{\text { Tan }}^{1}{ }^{1 B B S}$, Reon Yew Zhou Chin ${ }^{1} M B B S$, Anne Lee ${ }^{1} B S c$, Adeliza Mutalib ${ }^{1} M S c$, Poh Lian $\underline{\operatorname{Lim}}{ }^{3,6}{ }_{M D}$

\begin{abstract}
Introduction: The use of novel mRNA platforms for COVID-19 vaccines raised concern about vaccine safety, especially in Asian populations that made up less than $10 \%$ of study populations in the pivotal vaccine trials used for emergency use authorisation. Vaccine safety issues also remain a concern in assessing the clinical risks and benefits of vaccine boosters, particularly in specific age groups or segments of the population. This study describes a vaccination exercise involving Asian military personnel, and the adverse reactions and safety events observed.

Methods: Minor adverse reactions, hospitalisations and adverse events of special interest were monitored as part of the organisation's protocol for safety monitoring of COVID-19 vaccinations. All vaccine recipients were invited to complete an online adverse reaction questionnaire. Medical consults at the military's primary healthcare facilities were monitored for vaccine-related presentations. All hospitalisations involving vaccine recipients were analysed. Adverse reaction rates between doses, vaccines and age groups were compared.

Results: A total of 127,081 mRNA vaccine doses were administered to 64,661 individuals up to 24 July 2021. Common minor adverse reactions included fever/chills, body aches and injection site pain. These were more common after dose 2. Younger individuals experienced minor adverse reactions more frequently. Rare cases of anaphylaxis, Bell's palsy and myocarditis/pericarditis were observed. No deaths occurred.

Conclusion: Minor adverse reactions were less common than reported in other studies, and rates of anaphylaxis, Bell's palsy and myocarditis/pericarditis were comparable. Our study supports the favourable safety profile of mRNA COVID-19 vaccines, which may help guide decisions about booster doses if required.
\end{abstract}

Ann Acad Med Singap 2021;50(11)

Keywords: COVID-19, mRNA vaccine, public health, vaccine, vaccine safety

\section{INTRODUCTION}

The COVID-19 pandemic has led to significant changes in the way of life for many around the world. ${ }^{1,2}$ After more than a year, many countries continue to struggle with rising infection rates, and economic and social impact of the pandemic. ${ }^{3-5}$ COVID-19 vaccines have become available since the end of 2020, and are a critical part of the multipronged approach towards the resumption of international travel and trade, as well as the easing of societal restrictions. Singapore was one of the first countries to roll out COVID-19 vaccinations, beginning on 30 December 2020 with healthcare workers, using the mRNA COVID-19 vaccine manufactured by Pfizer-BioNTech, and subsequently adding the Moderna vaccine.

Although mRNA vaccines have been under study for many years, ${ }^{6}$ none had undergone clinical trials for infectious diseases until the COVID-19 pandemic. Interim analyses of phase 3 clinical trials assessing the efficacy, safety and tolerability of the Pfizer-BioNTech ${ }^{7}$

\footnotetext{
${ }^{1}$ Headquarters Medical Corps, Singapore Armed Forces, Singapore

${ }^{2}$ Department of Medicine, National University Health System, Singapore

${ }^{3}$ Saw Swee Hock School of Public Health, National University of Singapore, Singapore

${ }^{4}$ Department of Internal Medicine, Singapore General Hospital, Singapore

${ }^{5}$ National University Health System, Singapore

${ }^{6}$ National Centre for Infectious Diseases, Singapore

Correspondence: Dr Clive Tan, Biodefence Centre, Force Health Group, Headquarters Medical Corps, Singapore Armed Forces, 701 Transit Road, Singapore 778910.

Email: clivetan@nus.edu.sg
} 


\section{CLINICAL IMPACT}

\section{What is New}

- Findings from this cohort of military personnel support the overall safety of the mRNA COVID-19 vaccines among young individuals, mostly between the ages of 20 and 30 .

- Adverse reactions are mostly minor and are within the reported ranges in the literature.

\section{Clinical Implications}

- Our study supports the favourable safety profile of the mRNA COVID-19 vaccines for young individuals in Singapore and provides reassuring data to support rapid rollout of mass vaccination to other Asian populations in this age category.

and Moderna ${ }^{8}$ COVID-19 vaccines were published in December 2020 and February 2021, respectively. Since then, many health authorities including the US Food and Drug Administration, European Medicines Agency, and Singapore's Health Sciences Authority have authorised these 2 mRNA vaccines for emergency use.

However, studies and trials involving the mRNA COVID-19 vaccines thus far have had limited representation by Asian ethnicities. In the pivotal trials for the Pfizer-BioNTech and Moderna vaccines, Asians only comprised $4.3 \%{ }^{7}$ and $4.6 \%^{8}$ of participants, respectively. Real-world effectiveness and pharmacovigilance studies of mRNA vaccines from Asian and military populations are similarly limited, with most studies being conducted in non-Asian countries. ${ }^{9-11}$ As mRNA vaccine use has increased outside controlled clinical research settings, pharmacovigilance monitoring has identified rarer adverse events such as myocarditis and pericarditis, ${ }^{11,12}$ which were not observed in the phase 3 clinical trials involving smaller cohorts. Multiple studies suggest that a paucity of safety data for specific subgroups, including Asians, contributes to vaccine hesitancy, ${ }^{13,14}$ with many concerned about side effects. ${ }^{15,16}$ Given that booster doses may be needed in future to tackle waning vaccine efficacy and the emergence of new SARS-CoV-2 variants, ${ }^{17}$ it is useful to describe the safety profile and adverse reactions of mRNA COVID-19 vaccines in Asians, so as to contribute to the medical literature and address such reservations. ${ }^{18,19}$ We describe here the safety and adverse event profile in a multiethnic Asian cohort in the Singapore military who received at least 1 dose of an mRNA COVID-19 vaccine.

\section{METHODS}

We prospectively monitored post-vaccination adverse reactions in a cohort of Singapore military personnel who have received at least 1 dose of either the PfizerBioNTech or Moderna COVID-19 vaccine. Data were collected from vaccine recipients, military doctors and existing force health surveillance frameworks. Firstly, all recipients received an automated text message 7 days after the first dose, requesting them to complete a 1-minute online questionnaire, with another automated reminder sent 10 days after the same dose. This was repeated for the second dose. Respondents were asked to indicate the adverse reactions that they had experienced following vaccination from a list of adverse reactions. (Questionnaire of Supplementary Appendix in online version of this article.) Cardiacrelated symptoms such as abnormal sensation of heart beat, chest tightness and difficulty breathing were added to the list on 14 June 2021 due to reports of myocarditis and pericarditis following mRNA COVID-19 vaccination in the global press and literature. Free-text entries for reports prior to 14 June 2021 were analysed for cardiac-related symptoms and were included in the analysis for these symptoms where relevant. Secondly, military doctors who reviewed individuals seeking medical attention for postvaccination adverse reactions were instructed to assign a specific disease code on the electronic medical record (EMR). Lastly, we utilised existing force health surveillance frameworks for adverse reaction monitoring. This included a reporting system whereby all hospitalisations involving military personnel were reported to the military's Biodefence Centre. Adverse reaction data collected via these 3 methods were compiled into a single database for analysis. All the authors vouch for the completeness of the data and the accuracy of the analysis.

Sex and ethnicity records were retrieved from the EMR; sex was self-reported via institution-wide human resource channels, and ethnicity was classified using Singapore's census protocols. ${ }^{20}$ For each reaction, we calculated incidence rates and rate ratios after dose 2 versus dose 1 for both mRNA vaccines together and separately, with 95\% confidence intervals (CI). Incidence rates between doses, vaccines and age groups were compared using multivariate logistic regression, adjusting for vaccine administered, dose, age, sex and ethnic group where appropriate. A 2-sided $P$ value of $<0.05$ was considered statistically significant. Descriptive analyses were performed for rarer events including hospitalisations, cases of allergic reactions, anaphylaxis, Bell's palsy and myocarditis/pericarditis. 
Analyses were performed using $\mathrm{R}$ version 4.1.0. As data were collected as part of COVID-19 vaccination safety monitoring, ethics board approval was not required.

\section{RESULTS}

The COVID-19 vaccination exercise in Singapore's military commenced on 14 January 2021 using mRNA COVID-19 vaccines. Second doses were administered between 21 and 56 days after first doses, using the same vaccine that was administered for the first dose. Adverse reaction reports received up to 3 August 2021 were analysed. This corresponds to vaccinations administered up to 24 July 2021.

\section{Vaccination progress and demographics}

A total of 127,081 mRNA COVID-19 vaccine doses were administered to 64,661 individuals up to 24 July 2021. Of these, 62,420 individuals $(96.5 \%)$ have been fully vaccinated with 2 doses of a mRNA vaccine, comprising more than $90 \%$ of all military personnel ${ }^{21}$ (Fig. 1). The cohort of vaccine recipients was predominantly young (median age 22 years, interquartile range $20-30)$, male $(92.1 \%)$ and of Chinese ethnicity (Chinese 79.3\%, Malay 7.4\%, Indian $8.4 \%$, others $2.4 \%$ and unknown $2.4 \%$ ). In the cohort, 37,367 individuals $(57.8 \%)$ received at least 1 dose of the Pfizer-BioNTech vaccine, while 27,294 (42.2\%) received at least 1 dose of the Moderna vaccine (Table 1).
A total of 70,905 responses from 45,582 individuals (55.8\% of vaccine doses) were analysed. Age, sex and ethnic distributions of individuals with adverse reaction reports were similar to those of the general cohort of vaccine recipients (median age 22 years, 93.7\% male, $82.0 \%$ Chinese). Distribution of vaccine administered was similar to the general cohort of vaccine recipients (60.3\% Pfizer-BioNTech vaccine). Of the total responses, 39,154 were received after dose 1 and 31,751 were received after dose 2 (Table 1).

A decreasing response rate was observed over time from the start of vaccination campaign until the cut-off date, from a monthly average of $72.5 \%$ in January to $52.6 \%$ in July. In contrast, the proportion of adverse reaction reports that indicated at least 1 adverse reaction increased over time, from a monthly average of $41.9 \%$ in January to $62.2 \%$ in July (Fig. 2).

\section{Minor adverse reactions}

Injection site pain (18.4\%), body aches (10.9\%) and fatigue $(10.8 \%)$ were the most common adverse reactions after dose 1 (Fig. 3). Fever/chills (32.6\%), body aches $(20.9 \%)$ and fatigue $(14.1 \%)$ represented the most common adverse reactions after dose 2 . (Table S1 in online Supplementary Appendix.)

In general, adverse reactions were more common after dose 2 versus dose 1 (59.4\% vs $44.4 \%$, adjusted rate ratio [RR] 1.34, 95\% CI 1.32-1.36). Specifically, the highest adjusted RRs were observed for fever/chills

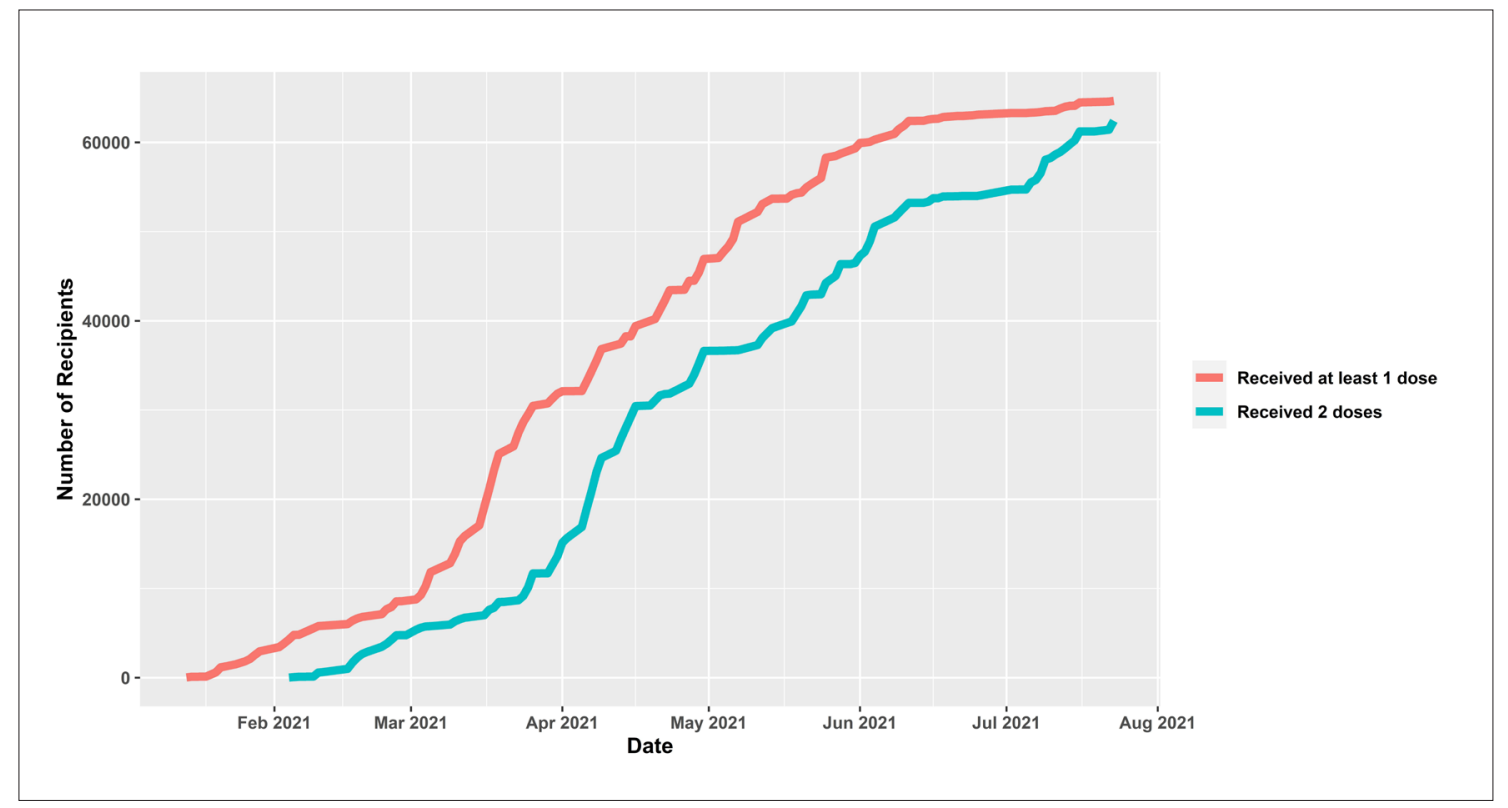

Fig. 1. Progress of vaccinations over time. (See online article for colour version.) 


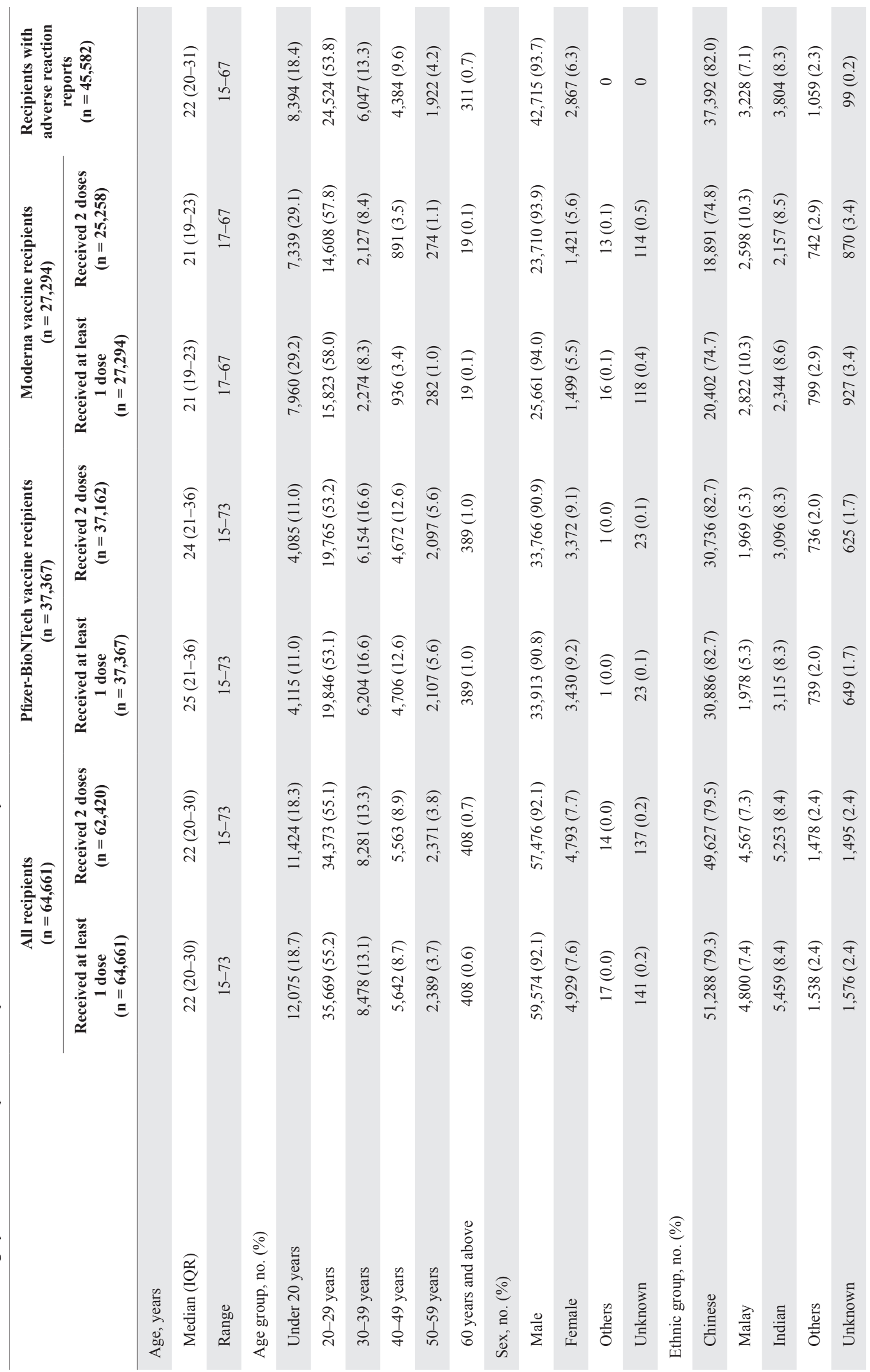




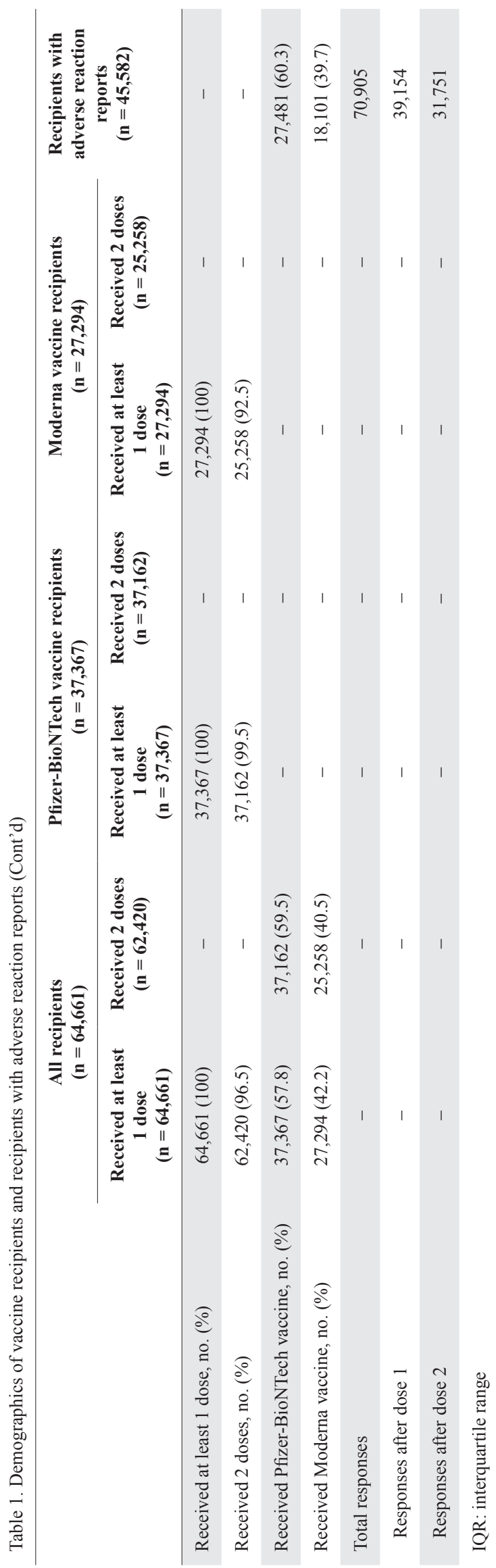

(RR 6.26, 95\% CI 6.00-6.54) and chest discomfort (RR 3.05, 95\% CI 2.27-4.11). Among adverse reactions that were less common after dose 2, injection site pain (RR $0.57,95 \%$ CI $0.55-0.59$ ) and muscle aches (RR $0.67,95 \%$ CI $0.62-0.72$ ) had the lowest adjusted RRs (Fig. 3). Trends for the more common adverse reactions remained consistent when analysed separately for each vaccine, except for fever/chills which was much more common in Moderna recipients (Tables S2-S4 in online Supplementary Appendix).

A smaller proportion of older recipients were observed to have experienced at least 1 adverse reaction, as compared to younger recipients (RR $0.62,95 \%$ CI $0.56-0.69$ for $\geq 60$ years compared to 20-29 years). This trend was consistent for body aches, fever/chills, headache and muscle aches (Fig. 4 and Table S5 in online Supplementary Appendix).

\section{Hospitalisations and deaths}

The median follow-up period was 119 days after dose 1 , and 104 days after dose 2. There was a total of 79 reports of hospitalisations, out of which 45 (57.0\%) occurred within 30 days of dose administration, 52 $(65.8 \%)$ within 42 days, and $61(77.2 \%)$ within 60 days. Case details of hospitalisations can be found in Table S6 of online Supplementary Appendix. Besides hospitalisations for perimyocarditis and chest pain with normal troponins, the number of hospitalisations for all other causes during our study period was similar to that during the same period in 2020. There were more hospitalisations for perimyocarditis and chest pain with normal troponins during our study period as compared to the same period in 2020 . There were no reports of deaths following COVID-19 vaccination.

\section{Allergic reactions and anaphylaxis}

Fifty-eight recipients (45.6 per 100,000 doses) were diagnosed with allergic reactions due to the mRNA COVID-19 vaccine after seeking medical attention for their adverse reactions. Thirty-four were diagnosed after dose 1, and 24 were diagnosed after dose 2 .

Of the 58, 2 were diagnosed with anaphylaxis due to the COVID-19 vaccine, both after dose 1 (rate: 1.6 per 100,000 doses).

The first case of anaphylaxis involves a 25 -year-old female with no known allergies. She reported chest tightness, shortness of breath, sensation of lip fullness, and hoarseness of voice 40 minutes after receiving her first dose. There were no abnormalities during the physical examination, including no observable lip 


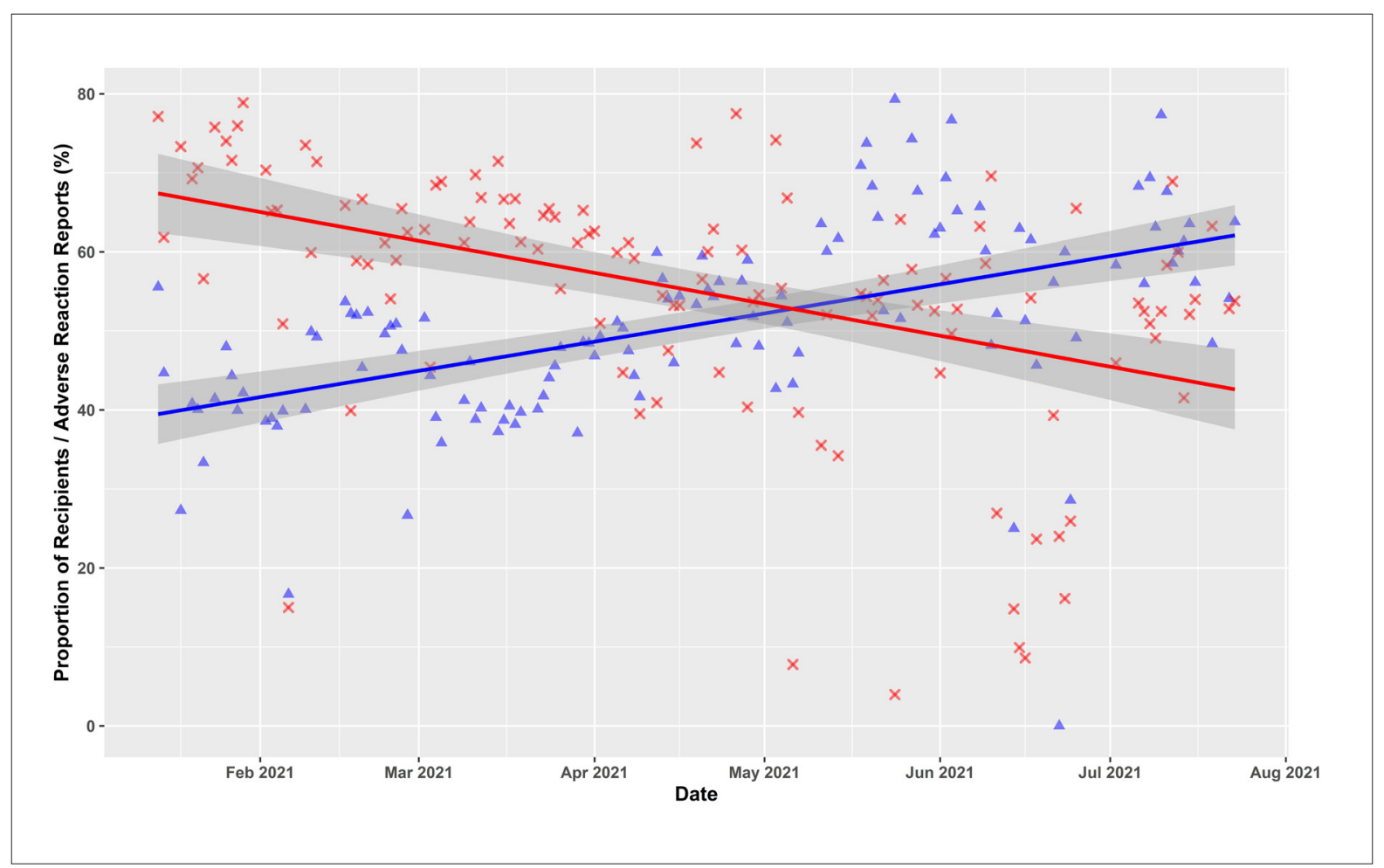

Fig. 2. Proportion of recipients with adverse reaction reports over time (red line) compared to proportion of adverse reaction reports with at least one adverse reaction over time (blue line). (See online article for colour version.)

Red crosses and blue triangles represent individual data points for each day during the vaccination exercise. Lines represent the line of best fit derived using a linear model. Grey areas represent $95 \%$ confidence intervals.

Red crosses: proportion of recipients with adverse reaction report for each day.

Blue triangles: proportion of adverse reaction reports with at least one adverse reaction for each day.

swelling. Her vital parameters were stable. Intramuscular adrenaline and intravenous hydrocortisone were administered, with subsequent improvement in her symptoms. She was monitored and discharged after a few hours.

The second case involves a 22-year-old male with a history of rash after seafood consumption. He reported rashes and giddiness within 30 minutes after his first dose, and subsequently developed nausea, diarrhoea, lethargy, shortness of breath, a globus sensation in his throat, and voice changes. He was given intravenous diphenhydramine and hydrocortisone, with symptomatic improvement. He was monitored and discharged after several hours.

\section{Bell's palsy}

One recipient was diagnosed with Bell's palsy after receiving dose 1 of the mRNA COVID-19 vaccine (rate: 0.8 per 100,000 doses). He is a 38 -year-old male with no past medical history. He developed left-sided facial numbness, hypogeusia, an asymmetrical smile, and drooling from the left side of his mouth 10 hours after vaccination. He was given a short course of prednisolone, and recovered fully after a few months.

\section{Myocarditis and pericarditis}

Three recipients were diagnosed with myocarditis/ pericarditis, all after receiving dose 2 of the mRNA COVID-19 vaccine (rate: 2.4 per 100,000 doses). All 3 had no history of cardiac conditions.

The first case involves an 18-year-old male who reported non-exertional chest discomfort and fever 1 day after his second dose. Electrocardiogram (ECG) showed pericarditic changes. Serum troponin was elevated. A transthoracic echocardiogram showed a preserved ejection fraction (EF) with no abnormalities or evidence of pericardial effusion. He was given a short course of colchicine and ibuprofen, and his symptoms had resolved completely.

The second case involves a 21-year-old male who reported worsening of pre-existing back discomfort with radiation to his chest, lethargy, and fever 4 days after his second dose. ECG showed pericarditic changes and serum troponin was elevated. Magnetic resonance 
Table 2. Details of cases of anaphylaxis, Bell's palsy and myocarditis/pericarditis

\begin{tabular}{|c|c|c|c|c|c|c|c|c|c|}
\hline $\begin{array}{l}\text { Age at } \\
\text { dose } 1\end{array}$ & Sex & $\begin{array}{l}\text { Ethnic } \\
\text { group }\end{array}$ & $\begin{array}{l}\text { Past medical/ } \\
\text { allergic } \\
\text { history }\end{array}$ & Vaccine & Dose & Clinical features & $\begin{array}{l}\text { Time/Day } \\
\text { of symptom } \\
\text { onset }\end{array}$ & Outcome & $\begin{array}{l}\text { Received } \\
\text { dose } 2\end{array}$ \\
\hline \multicolumn{10}{|c|}{ Anaphylaxis } \\
\hline 25 years & Female & Chinese & $\begin{array}{l}\text { No significant } \\
\text { history }\end{array}$ & $\begin{array}{l}\text { Pfizer- } \\
\text { BioNTech }\end{array}$ & 1 & $\begin{array}{l}\text { Chest tightness, shortness } \\
\text { of breath, subjective lip } \\
\text { fullness }\end{array}$ & 40 minutes & $\begin{array}{l}\text { Alive; fully } \\
\text { recovered }\end{array}$ & No \\
\hline 22 years & Male & Chinese & $\begin{array}{l}\text { Seafood } \\
\text { allergy }\end{array}$ & Moderna & 1 & $\begin{array}{l}\text { Generalised pruritus, } \\
\text { rash, nausea, diarrhoea, } \\
\text { lethargy, giddiness, } \\
\text { shortness of breath, } \\
\text { globus sensation in throat, } \\
\text { change in voice }\end{array}$ & $\begin{array}{l}\text { Within } 30 \\
\text { minutes }\end{array}$ & $\begin{array}{l}\text { Alive; fully } \\
\text { recovered }\end{array}$ & No \\
\hline \multicolumn{10}{|c|}{ Bell's palsy } \\
\hline 38 years & Male & Chinese & $\begin{array}{l}\text { No significant } \\
\text { history }\end{array}$ & $\begin{array}{l}\text { Pfizer- } \\
\text { BioNTech }\end{array}$ & 1 & $\begin{array}{l}\text { Left-sided facial } \\
\text { numbness, tongue } \\
\text { tingling, reduced taste } \\
\text { sensation } \\
\text { Left eye unable to close } \\
\text { completely, asymmetrical } \\
\text { smile, drooling from left } \\
\text { side of mouth }\end{array}$ & 9 hours & $\begin{array}{l}\text { Alive; fully } \\
\text { recovered }\end{array}$ & No \\
\hline \multicolumn{10}{|c|}{ Myocarditis/Pericarditis } \\
\hline 18 years & Male & Chinese & $\begin{array}{l}\text { No significant } \\
\text { history }\end{array}$ & Moderna & 2 & $\begin{array}{l}\text { Non-exertional chest } \\
\text { discomfort, fever }\end{array}$ & 1 day & $\begin{array}{l}\text { Alive; fully } \\
\text { recovered }\end{array}$ & $\begin{array}{l}\text { Not } \\
\text { applicable }\end{array}$ \\
\hline 21 years & Male & Chinese & $\begin{array}{l}\text { No significant } \\
\text { history }\end{array}$ & Moderna & 2 & $\begin{array}{l}\text { Non-exertional chest } \\
\text { discomfort, fever, lethargy }\end{array}$ & 4 days & $\begin{array}{l}\text { Alive; fully } \\
\text { recovered }\end{array}$ & $\begin{array}{l}\text { Not } \\
\text { applicable }\end{array}$ \\
\hline
\end{tabular}

imaging (MRI) of his heart revealed findings meeting the Lake Louise criteria for myocarditis, with a preserved $\mathrm{EF}$ and no pericardial involvement. He was given a short course of colchicine and ibuprofen, and his symptoms had resolved completely.

The third case involves an 18-year-old male who reported chest tightness and shortness of breath 4 days after his second dose. ECG did not show significant abnormalities and serum troponin was elevated. MRI findings did not meet the criteria for myocarditis. His EF was preserved, and there was no pericardial involvement. He was diagnosed with perimyocarditis clinically. He was given a short course of colchicine and ibuprofen, and his symptoms had since resolved completely.

Details of the aforementioned cases can be found in Table 2.

\section{DISCUSSION}

The COVID-19 vaccination exercise began in the Singapore military on 14 January 2021 . An organisationwide vaccination exercise was carried out, with several vaccination centres set up in various parts of Singapore. This enabled the achievement of a vaccination rate above $90 \%$ by July $2021 .{ }^{21}$ As COVID-19 vaccination was and remains voluntary, there has been a concerted push from the start of the vaccination exercise to educate all personnel on the rationale, known side effects and safety profile of the mRNA COVID-19 vaccines. The intent was to answer any queries early, to address possible sources of vaccine hesitancy. Additionally, both active and passive surveillance systems were implemented to monitor the adverse reaction profile of the vaccines postvaccination, to facilitate more comprehensive ascertainment of the spectrum of adverse reactions. 


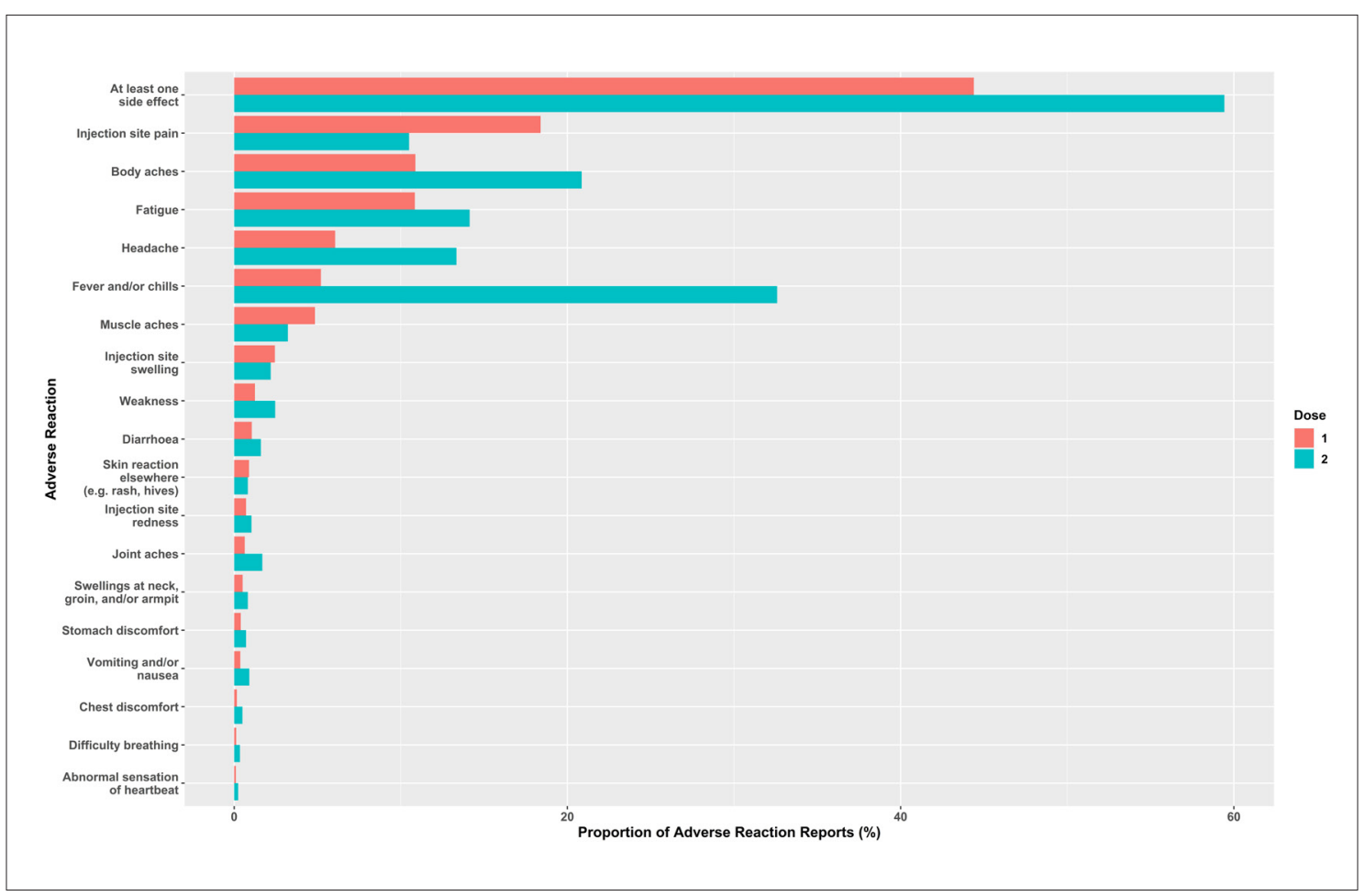

Fig. 3. Adverse reactions after dose 1 compared to dose 2. Adverse reactions are sorted by the proportion of dose 1 adverse reaction reports that reported the specified adverse reaction. (See online article for colour version.)

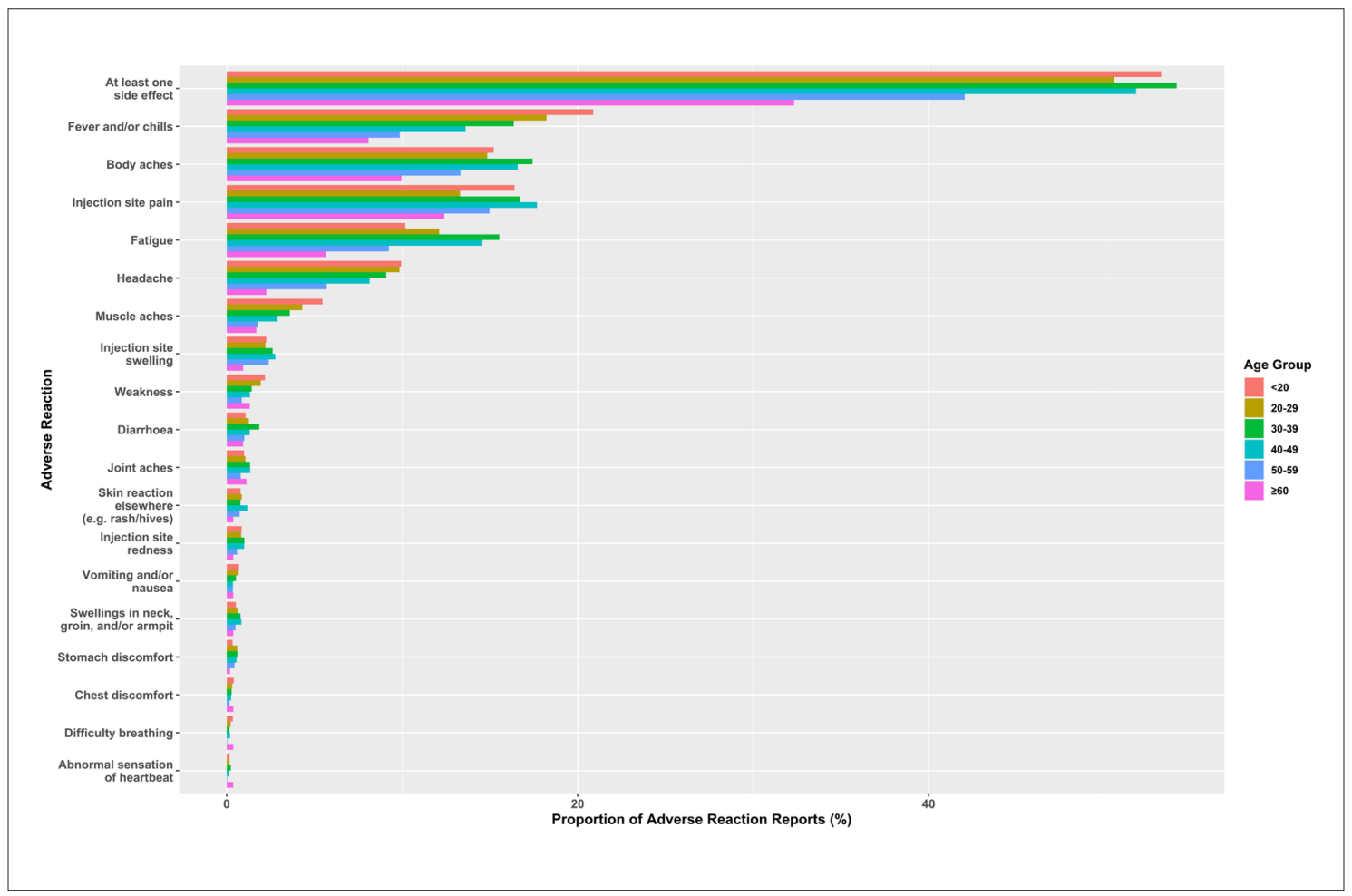

Fig. 4. Adverse reactions by age group. Adverse reactions are sorted by the proportion of respondents aged 20-29 years that reported the specified adverse reaction. (See online article for colour version.) 
The adverse reaction report response rate decreased with time, possibly due to increased familiarity with the vaccines and disinclination to provide nil returns. The proportion of reports with at least 1 adverse reaction increased over time, indicating that recipients with adverse reactions were reporting their reactions through the easily accessible reporting system. It is also possible that pervasive media messaging with the national vaccine rollout was raising awareness in vaccine recipients of adverse events from vaccination. While this suggests significant utility in a questionnaire-based self-reporting surveillance system, continued efforts to encourage questionnaire submission by vaccine recipients without adverse reactions are important to reduce non-response bias.

In our cohort of 64,661 individuals, the mRNA COVID-19 vaccines appear safe and well-tolerated. Post-vaccination adverse events were common: $44.4 \%$ and $59.4 \%$ of reports indicated at least 1 adverse reaction after dose 1 and dose 2, respectively. There were more adverse reactions reported after dose 2, consistent with higher reactogenicity. Our observations are comparable to phase 3 clinical trials of the mRNA COVID-19 vaccines, with body aches, fatigue, fever/ chills and injection site pain among the most common adverse reactions. ${ }^{7,8}$ We observed a generally lower rate of minor adverse reactions compared to those from studies conducted in the US, other countries and other populations. . $^{7,22,23}$ This was surprising as our cohort includes a larger proportion of younger individuals, for whom higher reactogenicity is expected..$^{22}$ Indeed, this trend is also seen in this study's subgroup analyses among the various age groups. Possible explanations for the lower rate in our study include differences in ethnicity, sex, health-seeking behaviour between study populations, and cultural differences in the perception and reporting of vaccine adverse reactions. ${ }^{24,25}$

Additionally, we observed a trend towards higher rates of minor adverse reactions among Moderna vaccine recipients as compared to Pfizer-BioNTech vaccine recipients. This has also been observed in other studies,${ }^{26}$ and may possibly reflect a stronger immune response produced by the vaccine. This hypothesis may be supported by recent studies observing higher effectiveness of the Moderna vaccine as compared to the Pfizer-BioNTech vaccine at 6 months after vaccination. ${ }^{27-29}$ Policymakers making decisions on the choice of a mRNA vaccine for vaccination exercises may need to weigh the possibly increased effectiveness of the Moderna vaccine needs against its possible increased risk of minor adverse reactions and reactogenicity.
There is a baseline incidence of medical events requiring hospital admissions, even in the absence of COVID-19 vaccination campaigns. The observed increase in hospitalisations for chest pain with normal troponins is likely due to a lower threshold for hospital referrals, with an increased awareness of postvaccination myocarditis among the general public and healthcare professionals. It is otherwise reassuring that the number of hospitalisations for other conditions were similar to that during the same period in 2020. The rates of anaphylaxis and myocarditis/pericarditis in our study are comparable to those reported in studies from other countries ${ }^{30-33}$ while our observed rate of Bell's palsy is lower. ${ }^{7,8}$ While these observations are reassuring and continue to support a favourable safety profile in favour of vaccinations for Asians, continued monitoring and pharmacovigilance is necessary in order to detect very low-frequency adverse events that may occur. ${ }^{34}$

This study has several strengths. Firstly, it provides useful and comprehensive data on adverse reactions in an Asian cohort that is predominantly male and relatively younger. Secondly, the large sample size, high questionnaire response rate, and comprehensive surveillance system for hospital admissions facilitated comprehensive capture of the full spectrum of adverse reactions.

The study also had several limitations. Firstly, there may be recall and non-response bias with passively collected self-reported data. Although the demographics of recipients with adverse reaction reports and the larger cohort of all recipients are similar, suggesting that non-response bias may be limited, factors not related to demographics may affect an individual's response. Secondly, participants could have altered their responses based on the perception that their military superiors might have access to this information, despite reassurances laid out in the survey. Thirdly, use of cross-sectional data collection may also miss events occurring after questionnaire submission. However, the overall vaccination safety monitoring system would capture later events if they were clinically significant. No such events were reported. Lastly, there are no data available for baseline rates of hospitalisation in a matched cohort, limiting the interpretation of our study's observed hospitalisation rates.

\section{CONCLUSION}

Our findings fill data gaps regarding the safety of the mRNA COVID-19 vaccines in Asians, and in young males. Most adverse reactions are mild, further emphasising the favourable safety profile of both the 
Pfizer-BioNTech and Moderna vaccines, regardless of ethnicity. Reliable information on vaccine safety in different settings and groups will continue to address vaccine hesitancy and guide organisations with vaccination rollouts, both in terms of primary courses and further doses if needed, so as to mitigate the economic, societal and health impact of COVID-19.

\section{Acknowledgement}

We thank Dr Hong Yee Lo (Singapore Armed Forces) for his advice and guidance with the development of vaccination policies and the safety monitoring plan for the vaccinations.

\section{REFEENCES}

1. Chen JIP, Yap JCH, Hsu LY, et al. COVID-19 and Singapore: From Early Response to Circuit Breaker. Ann Acad Med Singap 2020; 49:561-72

2. Tan THY, Toh MPHS, Vasoo S, et al. Coronavirus Disease 2019 (COVID-19): The Singapore Experience. A Review of the First Eight Months. Ann Acad Med Singap 2020;49:764-78.

3. McKibbin W, Fernando R. The economic impact of COVID-19. In: Economics in the Time of COVID-19. CEPR Press: London. 2020:45-51.

4. United Nations. Everyone Included: Social Impact of COVID-19. Available at: https://www.un.org/development/desa/dspd/everyoneincluded-covid-19.html. Accessed on 12 August 2021.

5. Ho RC, Tran BX, McIntyre RS. The impact of COVID-19 pandemic on global mental health: From the general public to healthcare workers. Ann Acad Med Singap 2021;50:198-9.

6. Pardi N, Hogan MJ, Porter FW, et al. mRNA vaccines - a new era in vaccinology. Nat Rev Drug Discov 2018;17:261-79.

7. Polack FP, Thomas SJ, Kitchin N, et al. Safety and Efficacy of the BNT162b2 mRNA Covid-19 Vaccine. N Engl J Med 2020;383:2603-15.

8. Baden LR, Sahly HME, Essink B, et al. Efficacy and safety of the mRNA-1273 SARS-CoV-2 Vaccine. N Engl Med 2021; 384:403-16.

9. Dagan N, Barda N, Kepten E, et al. BNT162b2 mRNA Covid-19 Vaccine in a Nationwide Mass Vaccination Setting. N Engl J Med 2021;384:1412-23.

10. Thompson MG, Burgess JL, Naleway AL, et al. Interim Estimates of Vaccine Effectiveness of BNT162b2 and mRNA-1273 COVID-19 Vaccines in Preventing SARS-CoV-2 Infection Among Health Care Personnel, First Responders, and Other Essential and Frontline Workers - Eight U.S. Locations, December 2020-March 2021. MMWR Morb Mortal Wkly Rep 2021; 70;495-500.

11. Montgomery J, Ryan M, Engler R, et al. Myocarditis Following Immunization With mRNA COVID-19 Vaccines in Members of the US Military. JAMA Cardiol 2021;6:1202-6.

12. Government of Israel. Surveillance of Myocarditis (Inflammation of the Heart Muscle) Cases Between December 2020 and May 2021 (Including). Available at: https://www.gov.il/en/departments/ news/01062021-03. Accessed on 21 August 2021.
13. Woolf K, McManus IC, Martin CA, et al. Ethnic differences in SARS-CoV-2 vaccine hesitancy in United Kingdom healthcare workers: Results from the UK-REACH prospective nationwide cohort study. Lancet Reg Health Eur 2021;100180.

14. Gadoth A, Halbrook M, Martin-Blais R, et al. Cross-sectional Assessment of COVID-19 Vaccine Acceptance Among Health Care Workers in Los Angeles. Ann Intern Med 2021;174:882-5.

15. Theis SR, Li PC, Kelly D, et al. Perceptions and Concerns Regarding COVID-19 Vaccination in a Military Base Population. Mil Med 2021;usab230

16. Park VT, Dougan M, Meyer O, et al. Differences in COVID-19 Vaccine Concerns Among Asian Americans and Pacific Islanders: The COMPASS Survey. J Racial Ethn Health Disparities 2021:1-13.

17. Nature. COVID vaccine boosters: the most important questions, 5 August 2021. Available at: https://www.nature.com/articles/d41586021-02158-6. Accessed on 12 August 2021.

18. Zhong BL, Luo W, Li HM, et al. Knowledge, attitudes, and practices towards COVID-19 among Chinese residents during the rapid rise period of the COVID-19 outbreak: a quick online cross-sectional survey. Int J Biol Sci 2020;16:1745-52.

19. Long V, Tan BYS, Tan LF. Knowledge and Perceptions of COVID-19 among the General Public in Singapore: A Cross-sectional Online Survey. Ann Acad Med Singap 2020; 49:1048-51.

20. Singapore Department of Statistics. Glossary of Terms and Definitions. In: Singapore Census of Population 2010 Statistical Release 1, 2011

21. Government of Singapore. Update on the SAF's COVID-19 Vaccination Management, 20 July 2021. Available at: https://www. mindef.gov.sg/web/portal/mindef/news-and-events/latest-releases/ article-detail/2021/July/20jul21_nr. Accessed on 12 August 2021.

22. Chapin-Bardales J, Gee J, Myers T. Reactogenicity Following Receipt of mRNA-Based COVID-19 Vaccines. JAMA 2021; 325:2201-2.

23. Health Sciences Authority. HSA's Safety Update \#5 COVID-19 Vaccines (30 December 2020 - 31 August 2021), 30 September 2021. Available at: https://www.hsa.gov.sg/COVID19-vaccines-safetyupdates. Accessed on 13 October 2021.

24. Gazerani P, Arendt-Nielsen L. The impact of ethnic differences in response to capsaicin-induced trigeminal sensitization. Pain 2005;117:223-9.

25. Kawi J, Reyes AT, Arenas RA. Exploring Pain Management Among Asian Immigrants with Chronic Pain: Self-Management and Resilience. J Immigr Minor Health 2019;21:1123-36.

26. Meo SA, Bukhari IA, Akram J, et al. COVID-19 vaccines: comparison of biological, pharmacological characteristics and adverse effects of Pfizer/BioNTech and Moderna Vaccines. Eur Rev Med Pharmacol Sci 2021;25:1663-9.

27. Reuters. Moderna says its COVID-19 shot $93 \%$ effective six months after second dose, 6 August 2021. Available at: https://www. reuters.com/business/healthcare-pharmaceuticals/moderna-saysits-covid-19-shot-remains-93-effective-4-6-months-after-seconddose-2021-08-05. Accessed on 12 August 2021.

28. WebMD. Pfizer Vaccine Protection Wanes After 6 Months Study Finds, 28 July 2021. Available at: https://www.webmd.com/vaccines/ covid-19-vaccine/news/20210728/pfizer-vaccine-protection-wanesafter-6-months-study-finds. Accessed on 12 August 2021.

29. Self WH, Tenforde MW, Rhoads JP, et al. Comparative Effectiveness of Moderna, Pfizer-BioNTech, and Janssen (Johnson \& Johnson) Vaccines in Preventing COVID-19 Hospitalizations Among Adults 
Without Immunocompromising Conditions - United States, March-August 2021. MMWR Morb Mortal Wkly Rep 2021; 70:1337-43.

30. Centers for Disease Control and Prevention COVID-19 Response Team and Food and Drug Administration. Allergic Reactions Including Anaphylaxis After Receipt of the First Dose of PfizerBioNTech COVID-19 Vaccine - United States, December 14-23, 15 January 2020. MMWR Morb Mortal Wkly Rep 2021;70:46-51.

31. Gargano JW, Wallace M, Hadler SC, et al. Use of mRNA COVID-19 Vaccine After Reports of Myocarditis Among Vaccine Recipients: Update from the Advisory Committee on Immunization
Practices - United States, June 2021. MMWR Morb Mortal Wkly Rep 2021;70:977-82.

32. Bozkurt B, Kamat I, Hotez PJ. Myocarditis With COVID-19 mRNA Vaccines. Circulation 2021;144:471-84.

33. Barda N, Dagan N, Ben-Shlomo Y, et al. Safety of the BNT162b2 mRNA Covid-19 Vaccine in a Nationwide Setting. N Engl J Med 2021;385:1078-90.

34. Schoch-Spana M, Brunson EK, Long R, et al. The public's role in COVID-19 vaccination: Human-centered recommendations to enhance pandemic vaccine awareness, access, and acceptance in the United States. Vaccine 2021;39:6004-12. 